\title{
Interaction effects on counting statistics and the transmission distribution
}

\author{
M. Kindermann ${ }^{1}$ and Yu. V. Nazarov ${ }^{2}$ \\ ${ }^{1}$ Instituut-Lorentz, Universiteit Leiden, P.O. Box 9506, 2300 RA Leiden, The Netherlands \\ ${ }^{2}$ Department of Nanoscience, Delft University of Technology, Lorentzweg 1, 2628 CJ Delft, The Netherlands
}

(Dated: April 2003)

\begin{abstract}
We investigate the effect of weak interactions on the full counting statistics of charge transfer through an arbitrary mesoscopic conductor. We show that the main effect can be incorporated into an energy dependence of the transmission eigenvalues and study this dependence in a nonperturbative approach. An unexpected result is that all mesoscopic conductors behave at low energies like either a single or a double tunnel junction, which divides them into two broad classes.
\end{abstract}

PACS numbers: 72.10.-d, 72.70.+m, 73.23.-b,

It has been shown that at low energy scales the relevant part of the electron-electron interaction in mesoscopic conductors comes from their electromagnetic environment [1]. The resulting dynamical Coulomb blockade has been thoroughly investigated for tunnel junctions [2]. The measure of the interaction strength is the external impedance $Z(\omega)$ at the frequency scale $\Omega=\max \left(e V, k_{B} T\right)$ determined by either the voltage $V$ at the conductor or its temperature $T$. If $z \equiv G_{Q} Z(\Omega) \ll 1$ (with the conductance quantum $G_{Q}=e^{2} / 2 \pi \hbar$ ) the interaction is weak, otherwise Coulomb effects strongly suppress electron transport.

A tunnel junction is the simplest mesoscopic conductor. An arbitrary mesoscopic conductor in the absence of interactions is characterized by its scattering matrix or, most conveniently, by a set of transmission eigenvalues $T_{n}$ 3]. This Landauer-Büttiker approach to mesoscopic transport can be extended to access the full counting statistics (FCS) of charge transfer [4]. The FCS contains not only the average current but also current noise and all higher moments of current correlations in a compact and elegant form.

Interaction effects on general mesoscopic conductors are difficult to quantify for arbitrary $z$. For $z \ll 1$, one can employ perturbation theory to first order in $z[5]$. Recent work 6, 7] associates the resulting interaction correction to the conductance with shot noise properties of the conductor. The interaction correction to noise is associated with the third cumulant of charge transfer [8]. This motivates us to study the interaction correction to all cumulants of charge transfer, i.e. to the FCS. The recent experiment [9] addresses the correction to the conductance at arbitrary transmission.

A tunnel junction in the presence of an electromagnetic environment exhibits an anomalous power-law I-V characteristic, $I(V) \simeq V^{2 z+1}$. The same power law behavior is typical for tunnel contacts between one-dimensional interacting electron systems, the so-called Luttinger liquids [10]. It has also been found for contacts with arbitrary transmission between single-channel conductors in the limit of weak interactions 11. In this case, the interactions have been found to renormalize the transmission.

In this Letter we study the effects of weak interactions $z \ll 1$ on the FCS of a phase coherent multi-channel conductor. In the energy range below the Thouless energy that we restrict our analysis to, its transmission probabilities $T_{n}$ are energy independent in the absence of interactions. We first analyze the interaction correction to first order in $z$. We identify an elastic and an inelastic contribution. The elastic contribution comes with a logarithmic factor that diverges at low energies suggesting that even weak interactions can suppress electron transport at sufficiently low energies. To quantify this we sum up interaction corrections to the FCS of all orders in $z$ by a renormalization group analysis. We show that the result is best understood as a renormalization of the transmission eigenvalues similar to that proposed in 11]. The renormalization brings about an energy dependence of the transmission eigenvalues according to the flow equation

$$
\frac{d T_{n}(E)}{d \ln E}=2 z T_{n}(E)\left[1-T_{n}(E)\right]
$$

To calculate transport properties in the presence of interactions, one evaluates $T_{n}(E)$ at the energy $E \simeq \Omega$.

With relation (11) we explore the effect of interactions on the distributions of transmission probabilities for various types of mesoscopic conductors. In general, their conductance $G$ and their noise properties display a complicated behavior at $z|\ln E| \simeq 1$ that depends on details of the conductor. However, in the limit of very low energies $z|\ln E| \gg 1$ we find only two possible scenarios. The first one is that the conductor behaves like a single tunnel junction with $G(V) \simeq V^{2 z}$. In the other scenario, the transmission distribution approaches that of a symmetric double tunnel junction. The conductance scales then as $G(V) \simeq V^{z}$. Any given conductor follows one of the two scenarios. This divides all mesoscopic conductors into two broad classes.

We start out by evaluating the interaction correction to the FCS to first order in $z$. We analyze a simple circuit that consists of a mesoscopic conductor in series with an external resistor $Z(\omega)$ biased with a voltage source $V$ (Fig. 1). For this we employ a non-equilibrium Keldysh action technique [12]. Within this approach, one represents the generating function $\mathcal{F}(\chi)$ of current fluctuations in the circuit as a path integral over the fields $\phi^{ \pm}(t)$ de- 


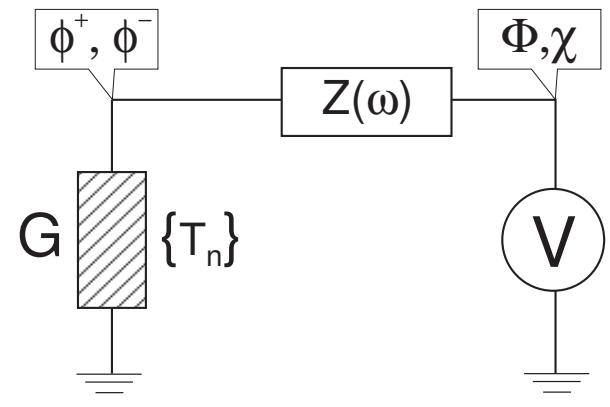

FIG. 1: Phase-coherent conductor (conductance $G$ ) in an electromagnetic environment (impedance $Z(\omega)$ ). We formulate the quantum dynamics of the system in terms of the fluctuating fields $\phi^{ \pm}(t)$.

fined on the time interval $[0, \tau]$, that represent the fluctuating voltage in the node shared by mesoscopic conductor and external resistor. The path integral representation of $\mathcal{F}(\chi)$ reads

$$
\begin{aligned}
\mathcal{F}(\chi)= & \int \mathcal{D} \phi^{+} \mathcal{D} \phi^{-} \exp \left\{-i \mathcal{S}_{\mathrm{c}}\left[\phi^{+}, \phi^{-}\right]\right. \\
& \left.-i \mathcal{S}_{\text {env }}\left[\Phi+\chi / 2-\phi^{+}, \Phi-\chi / 2-\phi^{-}\right]\right\}
\end{aligned}
$$

where $d \Phi(t) / d t \equiv e V$. Derivatives of $\mathcal{F}(\chi)$ with respect to $\chi$ at $\chi=0$ give the moments of the charge transferred through the circuit during a time interval of length $\tau$. The Keldysh action is a sum of two terms $\mathcal{S}_{\text {env }}$ and $\mathcal{S}_{\mathrm{c}}$ describing the environment and the mesoscopic conductor respectively.

We assume a linear electromagnetic environment that can be fully characterized by its impedance $Z(\omega)$ and its temperature $T$. Its action is bilinear in $\phi^{ \pm}$,

$$
\begin{aligned}
& \mathcal{S}_{\mathrm{env}}=\frac{1}{2 \pi} \int_{0}^{\tau} d t \int_{0}^{\tau} d t^{\prime}\left[\phi^{+}(t) A^{++}\left(t-t^{\prime}\right) \phi^{+}\left(t^{\prime}\right)\right. \\
& \left.+\phi^{+}(t) A^{+-}\left(t-t^{\prime}\right) \phi^{-}\left(t^{\prime}\right)+\phi^{-}(t) A^{--}\left(t-t^{\prime}\right) \phi^{-}\left(t^{\prime}\right)\right]
\end{aligned}
$$

with

$$
\begin{aligned}
& A^{++}(\omega)=-i \omega\left[z^{-1}(\omega)+2 N(\omega) \operatorname{Re} z^{-1}(\omega)\right] \\
& A^{+-}(\omega)=4 i \omega N(\omega) \operatorname{Re} z^{-1}(\omega) \\
& A^{--}(\omega)=-\left[A^{++}(\omega)\right]^{*}
\end{aligned}
$$

Here, $N(\omega) \equiv\left\{\exp \left[\omega / k_{B} T\right]-1\right\}^{-1}$ is the Bose-Einstein distribution function and $z(\omega)=G_{Q} Z(\omega)$ the dimensionless frequency-dependent impedance.

The action $\mathcal{S}_{\mathrm{c}}$ of the mesoscopic conductor can be expressed in terms of Keldysh Green functions $\check{G}_{R, L}$ (the "check" denotes $2 \times 2$ matrices in Keldysh space) of electrons in the two reservoirs adjacent to the conductor [13]. It takes the form of a trace over frequency and Keldysh indices,

$$
\mathcal{S}_{\mathrm{c}}=\frac{i}{2} \sum_{n} \operatorname{Tr} \ln \left[1+\frac{T_{n}}{4}\left(\left\{\check{G}_{\mathrm{L}}, \check{G}_{\mathrm{R}}\right\}-2\right)\right]
$$

and depends on the set of transmission eigenvalues $T_{n}$ that characterizes the conductor. The fields $\phi^{ \pm}(t)$ enter the expression as a gauge transform of $\check{G}$ in one of the reservoirs,

$$
\begin{aligned}
& \check{G}_{\mathrm{R}}=\check{G}^{\mathrm{res}} \text { and } \check{G}_{\mathrm{L}}\left(t, t^{\prime}\right)= \\
& {\left[\begin{array}{cc}
e^{i \phi^{+}(t)} & 0 \\
0 & e^{i \phi^{-}(t)}
\end{array}\right] \check{G}^{\mathrm{res}}\left(t-t^{\prime}\right)\left[\begin{array}{cc}
e^{-i \phi^{+}\left(t^{\prime}\right)} & 0 \\
0 & e^{-i \phi^{-}\left(t^{\prime}\right)}
\end{array}\right] .}
\end{aligned}
$$

$G^{\mathrm{res}}$ is the equilibrium Keldysh Green function

$$
\check{G}^{\mathrm{res}}(\epsilon)=\left(\begin{array}{cc}
1-2 f(\epsilon) & 2 f(\epsilon) \\
2[1-f(\epsilon)] & 2 f(\epsilon)-1
\end{array}\right),
$$

$f(\epsilon)$ being the equilibrium electron distribution function.

This defines our model that is valid for any external impedance but is hardly tractable in the general case. We proceed with perturbation theory in $z$ assuming that $z \ll 1$. To zeroth order in $z$ the fields $\phi^{ \pm}(t)$ do not fluctuate and are fixed to $e V t \pm \chi / 2$. Substituting this into Eq. (44) we recover Levitov's formula for non-interacting electrons,

$$
\begin{aligned}
& \ln \mathcal{F}^{(0)}(\chi) \equiv-i \tau \mathcal{S}^{(0)}(\chi)=\tau \int \frac{d \epsilon}{2 \pi} \sum_{n} \ln \{1 \\
& \left.+T_{n}\left[\left(e^{i \chi}-1\right) f_{\mathrm{L}}\left(1-f_{\mathrm{R}}\right)+\left(e^{-i \chi}-1\right) f_{\mathrm{R}}\left(1-f_{\mathrm{L}}\right)\right]\right\}
\end{aligned}
$$

$\left(f_{R} \equiv f\right.$ and $\left.f_{L}(\epsilon) \equiv f(\epsilon-e V)\right)$. Interaction effects manifest themselves at higher orders in $z$. To assess the first order correction, we expand the non-linear $\mathcal{S}_{\mathrm{c}}$ to second order in the fluctuating fields $\phi^{ \pm}(t)$. We integrate it over $\phi^{ \pm}$with the weight given by $\mathcal{S}_{\text {env }}$. The expression for the correction can be presented as [15]

$$
\begin{gathered}
\ln \mathcal{F}^{(1)}(\chi)=-i \tau \int_{0}^{\infty} d \omega \frac{\operatorname{Re} z(\omega)}{\omega}\left\{[2 N(\omega)+1] \mathcal{S}_{\mathrm{el}}^{(1)}(\chi)\right. \\
\left.+N(\omega) \mathcal{S}_{\text {in }}^{(1)}(\omega, \chi)+[N(\omega)+1] \mathcal{S}_{\text {in }}^{(1)}(-\omega, \chi)\right\}
\end{gathered}
$$

The three terms in square brackets correspond to elastic electron transfer, inelastic transfer with absorption of energy $\hbar \omega$ from the environment, and inelastic electron transfer with emission of this energy respectively. It is crucial to note that inelastic processes can only occur at frequencies $\omega \lesssim \Omega$ and that their contribution to the integral is thus restricted to this frequency range. In contrast, elastic contributions come primarily from frequencies exceeding the scale $\Omega$. If $z=\operatorname{const}(\omega)$ for $\omega \lesssim \Lambda$, the elastic correction diverges logarithmically, its magnitude being $\simeq z \ln \Lambda / \Omega$. This suggests that i. the elastic correction is more important than the inelastic one and ii. a small value of $z$ can be compensated for by a large logarithm, indicating the breakdown of perturbation theory. The upper cut-off energy $\Lambda$ is set either by the inverse 
$R C$-time of the environment circuit or the Thouless energy of the electrons in the mesoscopic conductor.

The concrete expression for $\mathcal{S}_{\text {in }}^{(1)}$ reads

$$
\begin{aligned}
\mathcal{S}_{\text {in }}^{(1)}(\omega, \chi)= & i \sum_{n} \int \frac{d \varepsilon}{2 \pi}\left\{D _ { n } D _ { n } ^ { + } \left[T_{n}\left(f_{L}-f_{L}^{+}\right)\right.\right. \\
& +2 T_{n}\left(e^{i \chi}-1\right) f_{L}\left(1-f_{R}^{+}\right) \\
& \left.+2 T_{n}^{2}(\cos \chi-1) f_{L}\left(1-f_{L}^{+}\right)\left(f_{R}^{+}-f_{R}\right)\right] \\
& \left.+T_{n} D_{n}+\left(1-D_{n}\right)\left(1-D_{n}^{+}\right)\right\} \\
& +\left\{\begin{array}{c}
\mathrm{R} \leftrightarrow \mathrm{L} \\
\chi \leftrightarrow-\chi
\end{array}\right\}
\end{aligned}
$$

where we have introduced the functions

$$
\begin{aligned}
D_{n}=\left\{1+T_{n}\right. & {\left[f_{L}\left(1-f_{R}\right)\left(e^{i \chi}-1\right)\right.} \\
+ & \left.\left.f_{R}\left(1-f_{L}\right)\left(e^{-i \chi}-1\right)\right]\right\}^{-1}
\end{aligned}
$$

and the notation

$$
f^{+}(\varepsilon)=f(\varepsilon+\omega), \quad D_{n}^{+}(\varepsilon)=D_{n}(\varepsilon+\omega) .
$$

We do not analyze $\mathcal{S}_{\text {in }}^{(1)}$ further in this Letter and instead turn to the analysis of the elastic correction.

It is important that the explicit form of the elastic correction can be presented as

$$
\begin{aligned}
\mathcal{S}_{\mathrm{el}} & =\sum_{n} \delta T_{n} \frac{\partial \mathcal{S}^{(0)}}{\partial T_{n}} \\
\text { with } \delta T_{n} & =-2 T_{n}\left(1-T_{n}\right) .
\end{aligned}
$$

This suggests that the main effect of interactions is to change the transmission coefficients $T_{n}$. It also suggests that we can go beyond perturbation theory by a renormalization group analysis that involves the $T_{n}$ only. In such an analysis one concentrates at each renormalization step on the "fast" components of $\phi^{ \pm}$with frequencies in a narrow interval $\delta \omega$ around the running cut-off frequency $E$. Integrating out these fields one obtains a new action for the "slow" fields. Subsequently one reduces $E$ by $\delta \omega$ and repeats the procedure until the running cut-off approaches $\Omega$. We find that at each step of renormalization the action indeed retains the form given by Eq. (4) and only the $T_{n}$ change, provided $z \ll \min \left\{1, G_{Q} / G\right\}$. The resulting energy dependence of the $T_{n}$ obeys Eq. (1). The strict proof of this involves manipulations on the action (4) with time dependent arguments $\phi^{ \pm}(t)$. It is very technical and we do not give it here. The approximations that we make in this renormalization procedure amount to a summation of the leading logarithms in every order of the perturbation series.

In the rest of the Letter we analyze the consequences of Eq. (1) for various mesoscopic conductors. Equation (1) can be explicitly integrated to obtain

$$
T_{n}(E)=\frac{\xi T_{n}^{\Lambda}}{1-T_{n}^{\Lambda}(1-\xi)}, \quad \xi \equiv\left(\frac{E}{\Lambda}\right)^{2 z}
$$

in terms of the "high energy" (non-interacting) transmission eigenvalues $T^{\Lambda}$. A mesoscopic conductor containing many transport channels is most conveniently characterized by the distribution $\rho_{\Lambda}(T)$ of its transmission eigenvalues [14]. It follows from Eq. (13) that the effective transmission distribution at the energy scale $E$ reads

$$
\rho_{E}(T)=\frac{\xi}{[\xi+T(1-\xi)]^{2}} \rho_{\Lambda}\left(\frac{T}{\xi+T(1-\xi)}\right) .
$$

We now analyze its low energy limit $\xi \rightarrow 0$. Any given transmission eigenvalue will approach zero in this limit. Seemingly this implies that for any conductor the transmission distribution approaches that of a tunnel junction, so that all $T_{n} \ll 1$. The overall conductance would be proportional to $\xi$ in accordance with Ref. [5].

Indeed, this is one of the possible scenarios. A remarkable exception is the case that the non-interacting $\rho_{\Lambda}$ has an inverse square-root singularity at $T \rightarrow 1$. Many mesoscopic conductors display this feature, most importantly diffusive ones [14]. In this case, the low-energy transmission distribution approaches a limiting function

$$
\rho_{*}(T) \propto \sqrt{\frac{\xi}{T^{3}(1-T)}} .
$$

The conductance scales like $\xi^{1 / 2} . \quad \rho_{*}$ is known to be the transmission distribution of a double tunnel junction: two identical tunnel junctions in series [16]. Indeed, one checks that for a double tunnel junction the form of the transmission distribution is unaffected by interactions. This sets an alternative low-energy scenario. We are not aware of transmission distributions that would give rise to other scenarios.

We believe that this is an important general result in the theory of quantum transport and suggest now a qualitative explanation. The statement is that the conductance of a phase-coherent conductor at low voltage and temperature $\Omega \ll \Lambda$ asymptotically obeys a power law with an exponent that generically takes two values,

$$
G \propto\left(\frac{\Omega}{\Lambda}\right)^{2 z}, \text { or } \quad G \propto\left(\frac{\Omega}{\Lambda}\right)^{z} .
$$

For tunneling electrons the exponent is $2 z$. An electron traverses the conductor in a single leap. The second possible exponent $z$ has been discussed in the literature as well, in connection with resonant tunneling through a double tunnel barrier in the presence of interactions [10]. This resonant tunneling takes place via intermediate discrete states contained between the two tunnel barriers. The halved exponent $\alpha=z$ occurs in the regime of the so-called successive electron tunneling. In this case, the electron first jumps over one of the barriers ending up in a discrete state. Only in a second jump over the second barrier the charge transfer is completed. Since it takes two jumps to transfer a charge, the electron feels only half the counter voltage due to interactions with electrons in the environmental impedance $Z$ at each hop. 

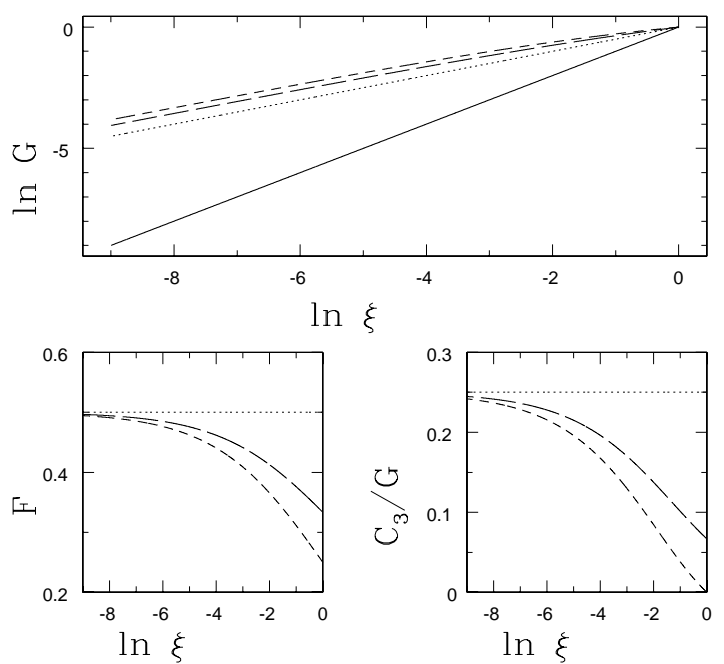

FIG. 2: Renormalization of conductance $G$ (logarithmically), Fano factor $F=C_{2} / G$ and third cumulant $C_{3}$ normalized by the conductance in a tunnel junction (solid line), a double tunnel barrier (dotted line), a double point contact (short dashed line), and a diffusive conductor (long lashed line).

Consequently, the exponent at each jump takes half the value for direct tunneling. Our results strongly suggest that this transport mechanism is not restricted to resonant tunneling systems, or, in other words, that resonant tunneling can occur in systems of a more generic nature than generally believed. As far as transport is concerned, a mesoscopic conductor is characterized by its scattering matrix regardless of the details of its inner structure. In this approach it is not even obvious that the conductor can accommodate discrete states. Nevertheless, the transmission distribution of this scattering matrix does depend on the internal structure of the conductor.
The inverse square root singularity of this distribution at $T \rightarrow 1$ for a double tunnel barrier is due to the formation of Fabry-Perot resonances between the two barriers. Probably similar resonances are at the origin of the same singularity for more complicated mesoscopic conductors with multiple scattering. They are then the intermediate discrete states that give rise to the modified scaling of the conductance in presence of interactions. One may speculate that in diffusive conductors these resonances are the so-called "nearly localized states" found in 17.

From equation (13) one concludes that the resonant tunneling scaling holds only if $G(E) \gg G_{Q}$ so that many transport channels contribute to the conductance. At sufficiently small energies, $G(E)$ becomes of the order of $G_{Q}$. All transmission eigenvalues are then small and the conductance crosses over to the tunneling scaling.

With Eqs. (13) and (14) we can evaluate the transmissions and the FCS in the intermediate regime $\xi \sim 1$. Fig. 2 shows the results for the first three cumulants of charge transfer $C_{n}$ for several types of conductors whose noninteracting transmission distributions $\rho_{\Lambda}(T)$ are known. Apart from the tunnel contact all these conductors approach the resonant tunneling scaling with noise properties of a double tunnel barrier at very small bias voltage $(\xi \ll 1)$.

We remark, that Eq. (12) generalizes the statements made in [7, 8] : At zero temperature, the interaction correction to the $\mathrm{n}$-th cumulant of transferred charge is proportional to the $(\mathrm{n}+1)$-th cumulant.

To conclude, we have investigated the effects of interactions on the FCS of a Landauer-Büttiker conductor and found that their main effect can be incorporated into an energy dependence of the transmission eigenvalues. For an arbitrary conductor, the conductance in the low-energy limit obeys one of two generic scaling laws.

The authors acknowledge useful discussions with C. W. J. Beenakker, K. B. Efetov, D. Esteve, L. I. Glazman, L. S. Levitov, and A. D. Zaikin. This work was supported by the Dutch Science Foundation NWO/FOM.
[1] Yu. V. Nazarov, Zh. Eksp. Teor. Fiz. 95, 975 (1989); G.L. Ingold and $\mathrm{Yu}$. V. Nazarov, in Single Charge Tunneling, edited by H. Grabert and M. H. Devoret, NATO ASI Series B294 (Plenum, New York, 1992).

[2] M. H. Devoret et. al. Phys. Rev. Lett. 64, 1824 (1990).

[3] C. W. J. Beenakker, Rev. Mod. Phys. 69, 731 (1997).

[4] L. S. Levitov and G. B. Lesovik, JETP Lett. 58, 230 (1993); cond-mat/9401004 L. S. Levitov et al. , J. Math. Phys. 37, 4845 (1996).

[5] Yu. V. Nazarov, Sol. St. Comm. 75, 669 (1990).

[6] D. S. Golubev, A. D. Zaikin, Phys. Rev. Lett. 86, 4887 (2001).

[7] A. Levy Yeyati et al., Phys. Rev. Lett. 87, 046802 (2001).

[8] A. V. Galaktionov et al. , cond-mat/0212494

[9] R. Cron et al., in Electronic Correlations: From Mesoto Nano-Physics, ed. by T. Martin, G. Montambaux, J. Trân Thanh Vân, EDP Sciences, 2001, p. 17.
[10] C. L. Kane and M. P. A. Fisher, Phys. Rev B 46, 15233 (1992).

[11] K. A. Matveev et al. , Phys. Rev. Lett. 71, 3351 (1993).

[12] M. Kindermann et al., cond-mat/0210617

[13] Y. V. Nazarov, Ann. Phys. (Leipzig) 8, 507 (1999).

[14] Yu. V. Nazarov, in: Quantum Dynamics of Submicron Structures, edited by H. A. Cerdeira, B. Kramer, G. Schön, Kluwer, 1995, p. 687; cond-mat/9410011

[15] We disregard corrections $\propto z(0)$ that account for the division of the voltage between mesoscopic conductor and external impedance (see [12] for details).

[16] J. A. Melsen and C. W. J. Beenakker, Physica B 203, 219 (1994).

[17] B. A. Muzykantskii and D. E. Khmelnitskii, Phys. Rev. B 51, 5480 (1995). 